\title{
Pneumatization of the sphenoid sinus, dorsum sellae and posterior clinoid processes in computed tomography
}

\author{
Wojciech Ilków ${ }^{1 \mathrm{~A}, \mathrm{~B}, \mathrm{C}, \mathrm{D}, \mathrm{E}, \mathrm{F}}$, Marek Waligóra ${ }^{2 \mathrm{~B}, \mathrm{C}}$, Marek Kunc ${ }^{3 \mathrm{D}, \mathrm{E}}$, Marek Kucharzewskii ${ }^{\mathrm{AEF}}$ \\ 'Department of Neurosurgery, University Teaching Hospital in Opole, Poland \\ 2Department of Medical Imaging, VITAL MEDIC, Kluczbork, Poland \\ ${ }^{3}$ Helimed Imaging Center, Opole, Poland \\ ${ }^{4} 5$ chool of Medicine with the Division of Dentistry in Zabrze, Department and Division of Descriptive and Topographic Anatomy, \\ Medical University of Silesia in Katowice, Zabrze Rokitnica, Poland
}

\section{Abstract}

Purpose: Assessment of sphenoid sinus (SS), posterior clinoid process (PCP), and dorsum sellae (DS) pneumatisation is significant when planning surgical approaches both to intra- and extrasellar pathologies. The authors analysed pneumatisation of the SS, with particular attention paid to pneumatisation of the PCP and DS.

Material and methods: The study was based on 100 computed tomography angiography examinations. SS, PCP, and DS pneumatisation grades were assessed using the classification system proposed by Hardy. The analyses were conducted in sagittal planes: midline plane (MP), sagittal posterior clinoid plane right (SPCP-R), and sagittal posterior clinoid plane left (SPCP-L). The occurrence of the highest pneumatisation grade (IVB according to Hardy), which encompasses DS and PCP, prompted the authors to conduct a further analysis in the coronal plane. In this way, seven DS and PCP pneumatisation symmetry types were identified.

Results: In the MP and SPCP-R, the most frequent pneumatisation grade was grade III ( $41 \%$ and $38 \%$, respectively). In the SPCP-L, grade IVA prevailed (41\%). Grade IVB was found in $12 \%$ of the SPCP-R images, $10 \%$ of the SPCP-L images, and in $12 \%$ of the MP images. Consistent pneumatisation grades in all analysed planes were found for $64 \%$ of cases. This was usually grade III noted in $28 \%$ of cases.

Conclusions: SS pneumatisation is characterised by considerable individual variability. There are a number of SS pneumatisation classification systems, but the system proposed by Hardy is the most useful for assessment of DS and PCP pneumatisation. Grade III of SS pneumatisation is the most common. Pneumatisation encompassing DS and PCP was found in $10-12 \%$ of cases.

Key words: posterior clinoid process, dorsum sellae, sphenoid sinus, pneumatisation, computed tomography.

\section{Introduction}

The sphenoid sinus (SS) is located within the body of the sphenoid bone, and its shape and size are characterised by considerable individual variability. The average SS volume is approximately $7.5 \mathrm{ml}$ in an adult. In cases of considerable pneumatisation, the SS can extend even to the fora- men magnum. Owing to its natural connection with the nasal cavity, the SS is significant when planning surgical approaches to both intra- and extrasellar pathologies [1]. The posterior clinoid process (PCP) and dorsum sellae (DS) are located in the middle cranial fossa on the superior surface of the sphenoid bone and form slight bony eminences of variable shapes and pneumatisation grades

Correspondence address:

Dr. Wojciech Ilków, 3 Łąkowa St., 46-060 Chrząszzzyce, Poland, e-mail: wojciechilkow@gmail.com

Authors' contribution:

A Study design · B Data collection · C Statistical analysis · D Data interpretation - E Manuscript preparation · F Literature search · G Funds collection 
[2-4]. In 1920, Congdon was the first to present a division of SS pneumatisation with reference to the sella turcica. He distinguished three types: I (conchal), II (presellar), and III (sellar) [5]. Later Hardy added types IVA and IVB to include more developed cases of pneumatisation.

The subject of SS pneumatisation is usually undertaken in the context of surgical approaches to intrasellar pathologies [6]. PCP and DS pneumatisation, on the other hand, is mentioned together with surgical accesses to pathologies located within the clivus or interpeduncular cistern [7,8].

Moreover, DS and PCP can form an obstacle by concealing deeper anatomic structures, for instance the basilar artery apex. In such cases, surgical conditions can be improved by reducing these structures (usually the posterior clinoidectomy $[\mathrm{PCP}]$ ) using a microdrill [9].

In the case of pneumatised DS or PCP removal (grade IVB according to Hardy), the risk of a dangerous complication in the form of cerebrospinal rhinorrhoea must be taken into account $[10,11]$.

In the case of extensive pneumatisation encompassing the DS and PCP, lesions in the clivus or intrapeduncular cistern can also be accessed through the trans-sphenoidal approach $[9,12]$.

There are a number of SS pneumatisation classification systems, but the system proposed by Hardy is the most useful for assessment of DS and PCP pneumatisation $[5,10]$.

The aim of this research was to develop reproducible and useful study methods to evaluate SS pneumatisation, with particular emphasis on DS and PCP pneumatisation grades.

\section{Material and methods}

The retrospective analysis involved anonymised computed tomography angiography (CT angiography) images of 100 patients ( 54 women and 46 men) aged $18-88$ years (mean age 52.49 years, standard deviation 18.64) diagnosed for headache. CT parameters were as follows: 16-row helical CT scanner GE BrightSpeed, collimation of $1 \mathrm{~mm}$, slice thickness of $0.625 \mathrm{~mm}$, pitch 1.0., voltage $120 \mathrm{kV}$. Non-ionic contrast material (Omnipaque 350) was injected into the one of the veins of the cubital region, i.e. the median cubital vein, at a rate of $3.5-4.5 \mathrm{ml} / \mathrm{s}$ to the total volume of $80-100 \mathrm{ml}$ using a power injector.

The study involved imaging scans encompassing all anatomic structures relevant for this investigation, without motion artefacts or foreign bodies, e.g. vascular clamps.

The study excluded patients:

- after craniocerebral trauma,

- after a neurosurgical procedure within the head,

- with a diagnosed intracranial tumour,

- with a pathology (tumour, inflammation etc.) within the SS.

SS pneumatisation in relation to the sella turcica, DS, and PCP was analysed in the sagittal plane using the classification system proposed by Hardy (Figure 1).

The analyses were carried out in the planes shown in Figure 2.

The occurrence of the highest pneumatisation grade, encompassing the DS and PCP (IVB according to Hardy), prompted the authors to conduct a further analysis in the coronal plane. In this way, seven pneumatisation symmetry types were identified within the DS and PCP (Figures 3 and 4).

This study complies with the current laws of Poland and it was approved by the Ethics Committee of the Medical University of Silesia in Katowice, Clinical Research Ref.: KNW/0022/KB/74/I/17.

\section{Statistical analysis}

Statistical calculations were performed in the PQStat version 1.6.2.901.

The relationship of sex and age with a pneumatisation grade was analysed using the $c^{2}$ test and Fisher's exact test.

The tested probability was deemed significant at $p<0.05$, and highly significant at $p<0.01$.
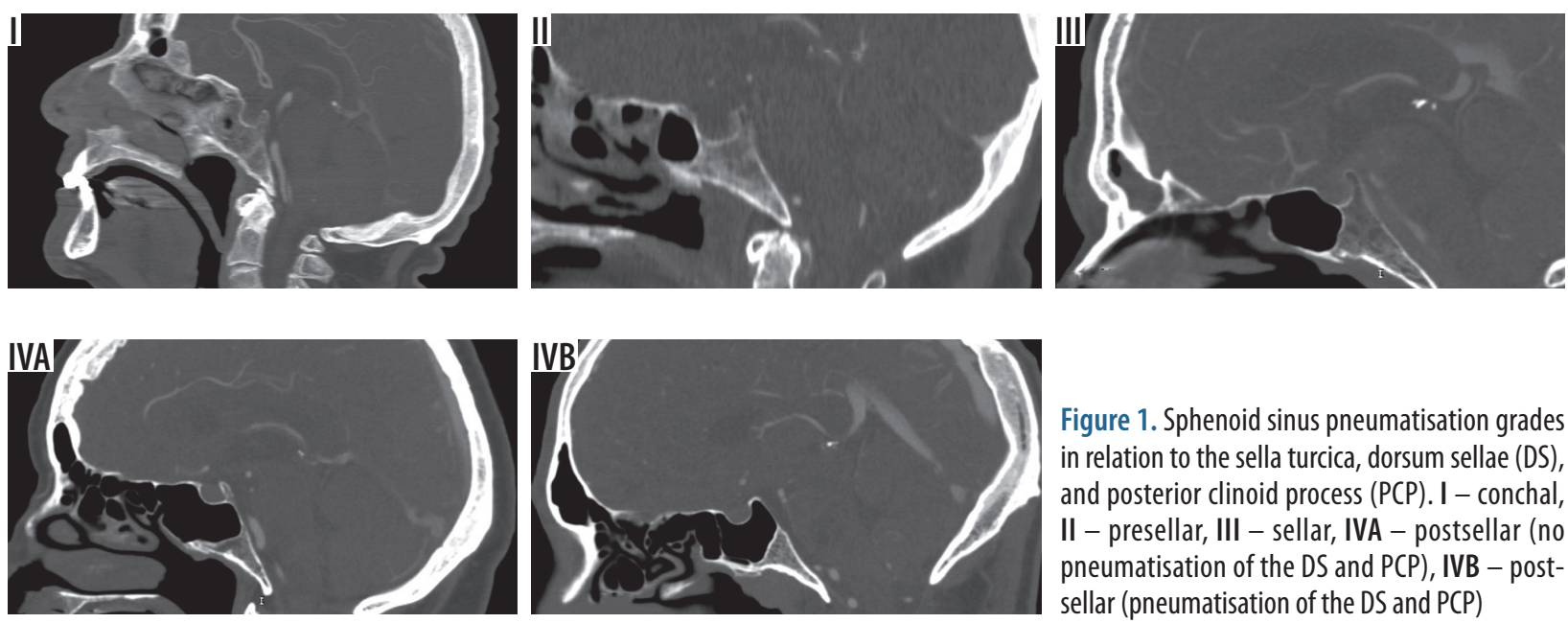

Figure 1. Sphenoid sinus pneumatisation grades in relation to the sella turcica, dorsum sellae (DS), and posterior clinoid process (PCP). I - conchal, II - presellar, III - sellar, IVA - postsellar (no pneumatisation of the DS and P(P), IVB - postsellar (pneumatisation of the DS and PCP) 

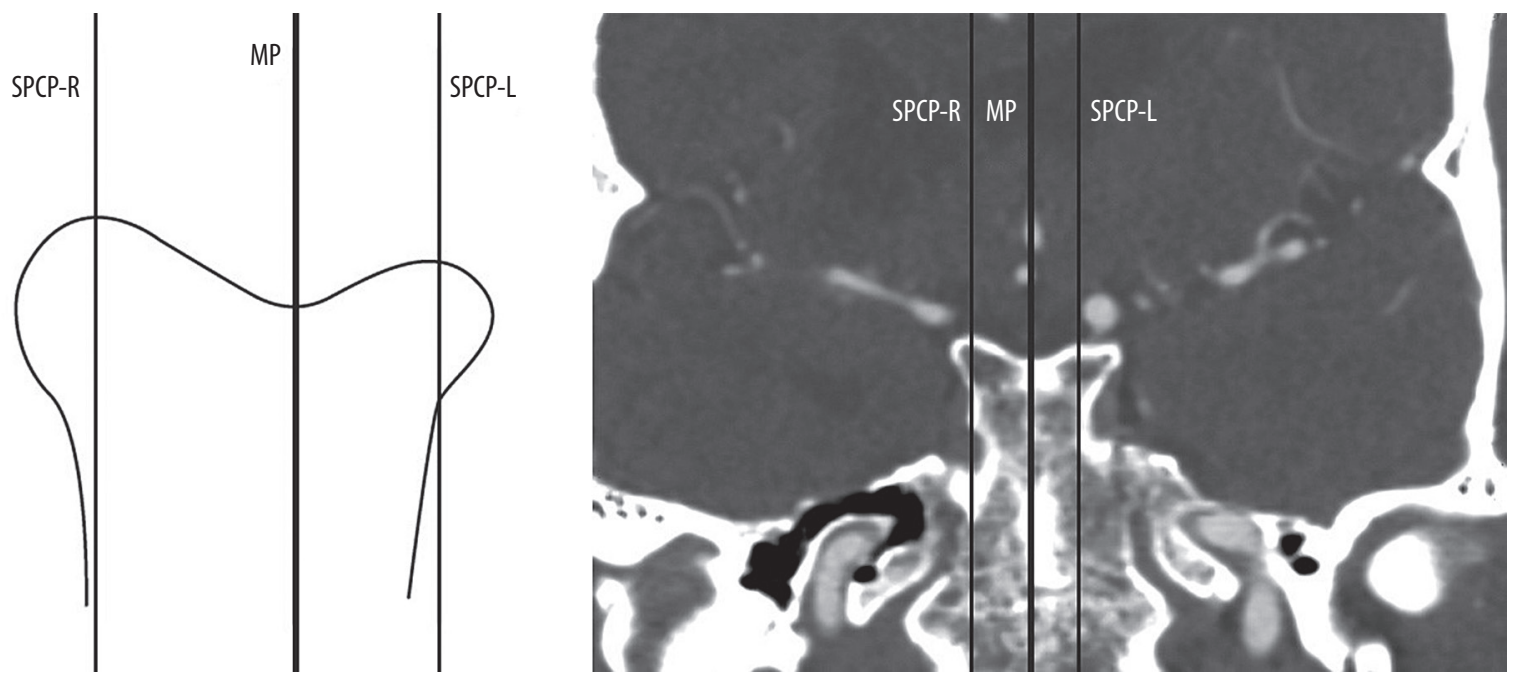

Figure 2. Planes for assessment of sphenoid sinus, dorsum sellae, and posterior clinoid process pneumatisation grades. MP - midline plane, SPCP-R - sagittal posterior clinoid plane right, SPCP-L - sagittal posterior clinoid plane left
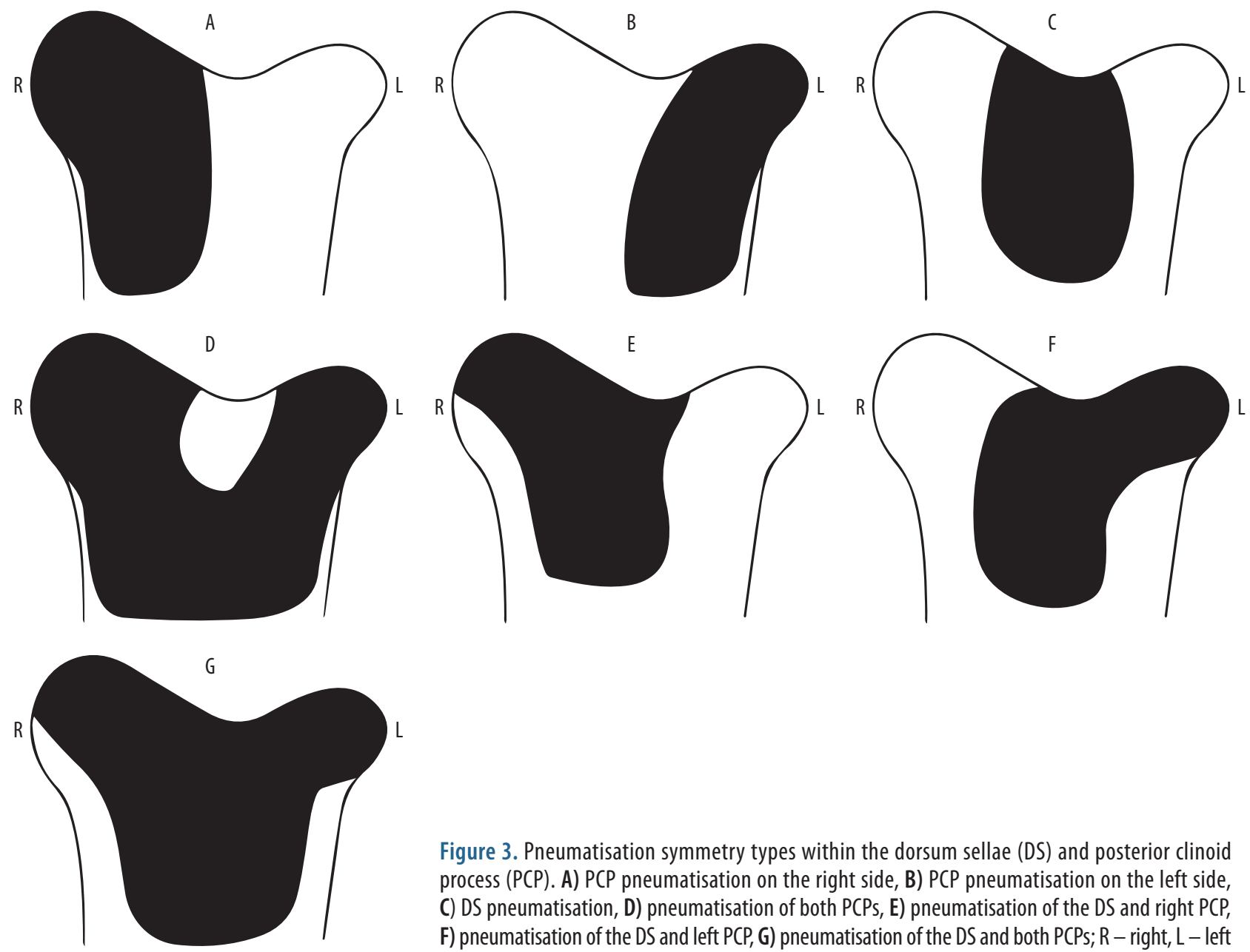

Figure 3. Pneumatisation symmetry types within the dorsum sellae (DS) and posterior clinoid process ( $P(P)$. A) $P(P$ pneumatisation on the right side, B) $P(P$ pneumatisation on the left side, C) DS pneumatisation, D) pneumatisation of both $P(P S, E)$ pneumatisation of the DS and right $P(P$, F) pneumatisation of the DS and left PCP, G) pneumatisation of the DS and both PCPs; $R$ - right, $L$ - left

\section{Results}

In the analysed material $(n=100)$, scans in the MP plane showed grade III pneumatisation in $41 \%$ of cases, which together with grade IVA, identified in $38 \%$ of cases, accounted for $79 \%$. In the SPCP-R plane, grade III was the most frequent (38\%) while in the SPCP-L, grade IVA prevailed (41\%). Grade I was the rarest in MP, SPCP-R, and SPCP-L planes.

The highest pneumatisation grade, i.e. grade IVB, was found in $12 \%$ of the SPCP-R scans, $10 \%$ of the SPCP-L scans, and $12 \%$ of the MP scans (Table 1 ).

In women $(n=54)$, the most common pneumatisation grade in all planes was grade III. 


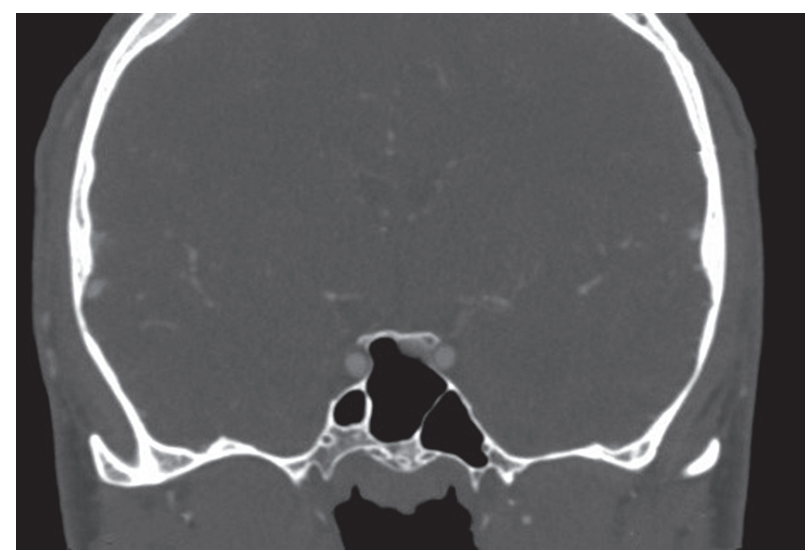

Figure 4. Pneumatisation symmetry type E within the dorsum sellae (DS) and posterior clinoid process (PCP)

Table 2. Pneumatisation grades in women

\begin{tabular}{|l|c|c|c|}
\hline \multirow{2}{*}{ Pneumatisation } & \multicolumn{3}{|c|}{ Plane } \\
\cline { 2 - 4 } & MP $(n=54)$ & SPCP-R $(n=54)$ & SPCP-L $(n=54)$ \\
\hline I & $3(6 \%)$ & $2(4 \%)$ & $2(4 \%)$ \\
\hline III & $2(4 \%)$ & $5(9 \%)$ & $6(11 \%)$ \\
\hline IVA & $23(42 \%)$ & $24(44 \%)$ & $22(40 \%)$ \\
\hline IVB & $23(42 \%)$ & $19(35 \%)$ & $21(39 \%)$ \\
\hline
\end{tabular}

I - conchal, II - presellar, III - sellar, IVA - postsellar - no pneumatisation of the dorsum sellae (DS) and posterior clinoid process (PCP), IVB - postsellar - pneumatisation of the DS and $P C P, M P$ - midline plane, SPCP-R - sagittal posterior clinoid plane right, SPCP-L - sagittal posterior clinoid plane left

Table 4. Consistent pneumatisation grades

\begin{tabular}{|l|c|c|c|}
$\begin{array}{l}\text { Consistent pneumatisation } \\
\text { grades in the MP/SPCP-R/SPCP-L }\end{array}$ & $\begin{array}{c}\text { Women } \\
(n=54)\end{array}$ & $\begin{array}{c}\text { Men } \\
(n=46)\end{array}$ & $\begin{array}{c}\text { Total } \\
(n=100)\end{array}$ \\
\hline I & $1(2 \%)$ & $0(0 \%)$ & $1(1 \%)$ \\
\hline II & $2(4 \%)$ & $2(5 \%)$ & $4(4 \%)$ \\
\hline III & $19(35 \%)$ & $9(19 \%)$ & $28(28 \%)$ \\
\hline IVA & $15(28 \%)$ & $11(24 \%)$ & $26(26 \%)$ \\
\hline IVB & $0(0 \%)$ & $5(11 \%)$ & $5(5 \%)$ \\
\hline
\end{tabular}

I - conchal, II - presellar, III - sellar, IVA - postsellar - no pneumatisation of the dorsum sellae (DS) and posterior clinoid process (PCP), IVB - postsellar - pneumatisation of the DS and $P C P, M P$ - midline plane, $S P C P-R$ - sagittal posterior clinoid plane right, SPCP-L - sagittal posterior clinoid plane left

The frequency of grade IVB pneumatisation in women in the SPCP-R plane was $8 \%$, in the SPCP-L plane $6 \%$, and in the MP plane $6 \%$ (Table 2).

In men $(n=46)$, the SPCP-R and SPCP-L images most frequently presented pneumatisation grade IVA: $37 \%$ and $43 \%$, respectively. In the MP plane, grade III prevailed: $39 \%$.

The frequency of grade IVB pneumatisation in men in the SPCP-R plane was $17 \%$, in the SPCP-L plane $15 \%$, and in the MP plane $19 \%$.

There were no cases of grade I pneumatisation in men (Table 3).
Table 1. Pneumatisation grades in all analysed planes

\begin{tabular}{|l|c|c|c|}
\hline \multirow{2}{*}{ Pneumatisation } & \multicolumn{3}{|c|}{ Plane } \\
\cline { 2 - 4 } & $\begin{array}{c}\text { MP } \\
(n=100)\end{array}$ & $\begin{array}{c}\text { SPCP-R } \\
(n=100)\end{array}$ & $\begin{array}{c}\text { SPCP-L } \\
(n=100)\end{array}$ \\
\hline I & $3(3 \%)$ & $2(2 \%)$ & $2(2 \%)$ \\
\hline II & $6(6 \%)$ & $12(12 \%)$ & $11(11 \%)$ \\
\hline III & $41(41 \%)$ & $38(38 \%)$ & $36(36 \%)$ \\
\hline IVB & $38(38 \%)$ & $36(36 \%)$ & $41(41 \%)$ \\
\hline
\end{tabular}

I - conchal, II - presellar, III - sellar, IVA - postsellar - no pneumatisation of the dorsum sellae (DS) and posterior clinoid process (PCP), IVB - postsellar - pneumatisation of the DS and PCP, MP - midline plane, SPCP-R - sagittal posterior clinoid plane right, SPCP-L - sagittal posterior clinoid plane left

Table 3. Pneumatisation grades in men

\begin{tabular}{|l|c|c|c|}
\hline \multirow{2}{*}{ Pneumatisation } & \multicolumn{3}{|c|}{ Plane } \\
\cline { 2 - 4 } & MP $(n=46)$ & SPCP-R $(n=46)$ & SPCP-L $(n=46)$ \\
\hline I & $0(0 \%)$ & $0(0 \%)$ & $0(0 \%)$ \\
\hline II & $4(9 \%)$ & $7(15 \%)$ & $5(11 \%)$ \\
\hline III & $18(39 \%)$ & $14(31 \%)$ & $14(31 \%)$ \\
\hline IVA & $15(33 \%)$ & $17(37 \%)$ & $20(43 \%)$ \\
\hline
\end{tabular}

I - conchal, II - presellar, III - sellar, IVA - postsellar - no pneumatisation of the dorsum sellae (DS) and posterior clinoid process (PCP), IVB - postsellar - pneumatization of the DS and PCP, MP - midline plane, SPCP-R - sagittal posterior clinoid plane right, SPCP-L - sagittal posterior clinoid plane left

Table 5. Type of pneumatisation symmetry within the dorsum sellae (DS) and posterior clinoid process $(P C P)$

\begin{tabular}{|l|c|c|c|}
$\begin{array}{l}\text { Type of pneumatisation } \\
\text { symmetry within the DS and PCP }\end{array}$ & $\begin{array}{c}\text { Women } \\
(n=54)\end{array}$ & $\begin{array}{c}\text { Men } \\
(n=46)\end{array}$ & $\begin{array}{c}\text { Total } \\
(n=100)\end{array}$ \\
\hline A & $0(0 \%)$ & $3(7 \%)$ & $3(3 \%)$ \\
\hline B & $3(6 \%)$ & $0(0 \%)$ & $3(3 \%)$ \\
\hline C & $0(0 \%)$ & $1(2 \%)$ & $1(1 \%)$ \\
\hline D & $0(0 \%)$ & $0(0 \%)$ & $0(0 \%)$ \\
\hline E & $3(6 \%)$ & $1(2 \%)$ & $4(4 \%)$ \\
\hline F & $0(0 \%)$ & $2(4 \%)$ & $2(2 \%)$ \\
\hline G & $0(0 \%)$ & $5(11 \%)$ & $5(5 \%)$ \\
\hline
\end{tabular}

There were no significant $(p>0.05)$ correlations between pneumatisation grades and sex or age of the patients.

In this material $(n=100)$, consistent pneumatisation grades for all planes (MP, SPCP-R, SPCP-L) were found for $64 \%$ of cases. Grade III was the most common: in $28 \%$ of cases (19 women and nine men), while grade I was the rarest: in $1 \%$ (only one female patient) (Table 4 ).

In this material $(n=100)$, pneumatisation symmetry type A within the DS and PCP was found in men only (3\% of cases), whereas type B was present only in women (3\% of cases). 
Type G was the most common (5\% of cases) and occurred only in men. There were no cases of type D (Table 5).

\section{Discussion}

The SS is a pneumatic space located within the body of the sphenoid bone. Its size and shape are variable, and it is lined with mucous membrane and divided with one or several vertical septa [1].

It is formed in the fourth month of the foetal development from the nasal capsule of the embryonic nose [1].

At birth, the SS is not fully developed, shows no signs of pneumatisation, and its diameter reaches approximately $2 \mathrm{~mm}$ [13].

SS pneumatisation progresses with age. It reaches the sella turcica at approximately seven years of age and ends during puberty $[1,13,14]$.

SS agenesis is seen in rare cases (1-1.5\%), and no progress of the SS pneumatisation process up to the age of 10 years may indicate a pathology within the SS $[1,15]$.

SS pneumatisation is an interesting subject for numerous fields of medicine (neurosurgery, laryngology, maxillofacial surgery). Owing to the anatomic connection between the SS and the nasal cavity, the SS constitutes a route for an endoscope to reach different intra- and extrasellar pathologies [16].

According to Teatini et al., the SS is the most variable space in the human body [17]. That is why thorough analysis of the anatomy in this region prior to a surgery is very important. Due to its location in close proximity to various vital structures, such as the pituitary gland, the cavernous sinus, the optic nerve, or the internal carotid artery, extensive SS pneumatisation that encompasses the DS or PCP might lead to the thinning of its walls and secondary inclusion of these structures into the sinus. This entails the risk of their damage during a surgical procedure [17].

The initial division of SS pneumatisation proposed by Congdon was created mainly for the purposes of transsphenoidal access to intrasellar pathologies $[5,18]$.

Subsequently, as endoscopy developed and the transsphenoidal access was broadened to regions neighbouring the sella turcica, such as the clivus or suprasellar region, other classification systems that included the DS and PCP were developed, including the one proposed by Hardy.

Güldner et al. analysed CT scans of the paranasal sinuses in 580 patients ( 313 women and 267 men). They found conchal pneumatisation in two patients $(0.3 \%)$, presellar pneumatisation in 38 patients (6.6\%), sellar pneuma- tisation in 332 patients (57.2\%), as well as postsellar IVA pneumatization in $104(17.9 \%)$ and postsellar IVB pneumatisation also in 104 (17.9\%) patients [19].

Based on high-resolution computed tomography (HRCT) of the paranasal sinuses, Tomovic et al. analysed SS pneumatisation in 170 patients (96 women and 74 men). They found grade I pneumatisation in 1.8\% (three patients), grade II in $7.3 \%$ (12 patients), grade III in $47.6 \%$ (78 patients), and grade IV in $43 \%$ (71 patients) [20].

Idowu et al. conducted head CT scans in 60 patients ( 23 women and $37 \mathrm{men}$ ) and found that sellar pneumatisation was the most common (50 patients, $83 \%$ ). The authors found no cases of conchal pneumatisation [21].

The outcomes of the present study are similar to those reported by other authors. In 100 patients, MP images showed conchal grade I pneumatisation in three patients (3\%), presellar type II in six patients (6\%), sellar type III in 41 patients $(41 \%)$, and postsellar type IV in 50 patients (50\%): IVA in 38 and IVB in 12 patients.

For the most accurate assessment, the authors analysed SS pneumatisation in three sagittal planes (MP, SPCP-R, and SPCP-L). When classifying a case to the IVB group in the MP plane, the DS was considered completely pneumatised. When classifying a case to the IVB group in the SPCP-R or SPCP-L planes, the authors assumed that the right and left PCPs, respectively, were pneumatised completely. In this way, the frequency of right PCP pneumatisation was $12 \%$, left PCP pneumatisation $10 \%$, and DS pneumatisation $12 \%$.

Güldner et al. found no significant differences between the frequency of given SS pneumatisation types depending on gender [19]. Tomovic et al., on the other hand, showed no correlations between SS pneumatisation and age [20].

In the material analysed in the present study $(n=100)$ there were no statistically significant $(p>0.05)$ correlations between pneumatisation grades and sex or age of the patients.

\section{Conclusions}

SS pneumatisation is characterised by considerable individual variability. Pneumatisation encompassing the DS and PCP, i.e. Hardy IVB, was found in $10-12 \%$ of cases. Type IVB by Hardy includes seven types of pneumatisation.

\section{Conflict of interest}

The authors report no conflict of interest.

References

1. Cakur B, Sümbüllü MA, Yılmaz AB. A retrospective analysis of sphenoid sinus hypoplasia and agenesis using dental volumetric CT in Turkish individuals. Diagn Interv Radiol 2011; 17: 205-208.
2. Cheng Y, Chen Y, Zhou Z, et al. Anatomical study of posterior clinoid process (PCP) and its clinical meanings. J Craniofac Surg 2015; 26: $537-540$ 
3. Fernandez-Miranda JC, Gardner PA, Rastelli MM Jr, et al. Endoscopic endonasal transcavernous posterior clinoidectomy with interdural pituitary transposition. J Neurosurg 2014; 121: 91-99.

4. Youssef AS, van Loveren HR. Posterior clinoidectomy: dural tailoring technique and clinical application. Skull Base 2009; 19: 183-191.

5. Congdon ED. The distribution and mode of origin of septa and walls of the sphenoid sinus. Anat Rec 1920; 18: 97-123.

6. Hamid O, El Fiky L, Hassan O, et al. Anatomic variations of the sphenoid sinus and their impact on trans-sphenoid pituitary surgery. Skull Base 2008; 18: 9-15.

7. Tang CT, Baidya NB, Tseng KY, et al. Posterior clinoid process as a landmarker in current endoscopic-assisted neurosurgical approaches. Formosan Journal of Surgery 2012; 45: 45-50.

8. Tjahjadi M, Kivelev J, Serrone JC, et al. Factors determining surgical approaches to basilar bifurcation aneurysms and its surgical outcomes. Neurosurgery 2016; 78: 181-191.

9. Silva D, Attia M, Kandasamy J, et al. Endoscopic endonasal posterior clinoidectomy. Surg Neurol Int 2012; 3: 64.

10. Hardy J. Surgery of the pituitary gland, using the open trans-sphenoidal approach. Comparative study of 2 technical methods. Ann Chir 1967; 21: 1011-1022.

11. Štoković N, Trkulja V, Dumić-Čule I, et al. Sphenoid sinus types, dimensions and relationship with surrounding structures. Ann Anat 2016; 203: 69-76.

12. Lu Y, Pan J, Qi S, et al. Pneumatization of the sphenoid sinus in Chinese: the differences from Caucasian and its application in the extended transsphenoidal approach. J Anat 2011; 219: 132-142.
13. Charsoula A, Nalmpantidou C, Torounidis I, et al. Age-related CT appearance of sphenoid sinus in infants and children. ECR Poster 2011; No. C-1443; doi: 10.1594/ecr2011/C-1443.

14. Budu V, Mogoantă CA, Fănuță B, et al. The anatomical relations of the sphenoid sinus and their implications in sphenoid endoscopic surgery. Rom J Morphol Embryol 2013; 54: 13-16.

15. Anik I, Anik Y, Koc K, et al. Agenesis of sphenoid sinuses. Clin Anat 2005; 18: 217-219.

16. Wiebracht ND, Zimmer LA. Complex anatomy of the sphenoid sinus: a radiographic study and literature review. J Neurol Surg B Skull Base 2014; 75: 378-382.

17. Teatini G, Simonetti G, Salvolini U, et al. Computed tomography of the ethmoid labyrinth and adjacent structures. Ann Otol Rhinol Laryngol 1987; 96 (3 Pt 1): 239-250.

18. ELKammash TH, Enaba MM, Awadalla AM. Variability in sphenoid sinus pneumatization and its impact upon reduction of complications following sellar region surgeries. The Egyptian Journal of Radiology and Nuclear Medicine 2014; 45: 705-714.

19. Güldner C, Pistorius SM, Diogo I, et al. Analysis of pneumatization and neurovascular structures of the sphenoid sinus using cone-beam tomography (CBT). Acta Radiol 2012; 53: 214-219.

20. Tomovic S, Esmaeili A, Chan NJ, et al. High-resolution computed tomography analysis of variations of the sphenoid sinus. J Neurol Surg B Skull Base 2013; 74: 82-90.

21. Idowu OE, Balogun BO, Okoli CA. Dimensions, septation, and pattern of pneumatization of the sphenoidal sinus. Folia Morphol (Warsz) 2009; 68: 228-232. 